\title{
A Rare Case of Acrodermatitis Enteropathica in a One Year Old Child
}

\author{
Nilofer Ziauddin ${ }^{1}$, Nayeem Sadath Haneef ${ }^{2}$, Nikhat ${ }^{3}$ \\ ${ }^{1}$ Department of Dermatology Venereology and Leprosy, Deccan College of Medical Sciences, \\ Hyderabad, Telangana, India. ${ }^{2}$ Department of Dermatology Venereology and Leprosy, Deccan \\ College of Medical Sciences, Hyderabad, Telangana, India. ${ }^{3}$ Department of Dermatology \\ Venereology and Leprosy, Deccan College of Medical Sciences, Hyderabad, Telangana, India.
}

\section{INTRODUCTION}

Acrodermatitis enteropathica (AE) is a rare autosomal recessive disorder caused by zinc deficiency.[1] It can be classified as primary zinc deficiency, genetically based zinc deficiency (classical, AE, acquired zinc deficiency of lactogenic origin), and acquired secondary zinc deficiency. ${ }^{[2]}$ Genetic zinc deficiency is associated with the defects in two zinc transporters, of which one is involved in intestinal zinc uptake ZRT- and IRTlike Protein-4 (ZIP), causing classical and AE. The other is responsible for zinc secretion in breast milk zinc transporter-2 (ZnT) resulting in zinc deficiency of lactogenic origin. ${ }^{[3]}$ Here, we have discussed and reviewed the clinical aspects and probable role of zinc transporters in the manifestation of AE.

Acrodermatitis enteropathica (AE), manifests as acral and periorificial dermatitis, alopecia, intractable diarrhoea, and failure to thrive. It is classified as primary zinc deficiency, genetically based deficiency, and acquired secondary deficiency. We hereby report a case of genetically based $\mathrm{AE}$ in a one year old child. After reviewing the literature, we have also emphasized the possible role of genetics in the manifestation of AE.

\section{PRESENTATION OF CASE}

One-year old male child was brought with complaints of generalized weakness, irritability, loose stools from the past 20 days. Later child developed extensive skin erosions all over the body predominantly over the acral, periorificial, and over the joints, from the past 15 days. Loose stools were watery in consistency. There was a history of weaning from breast milk to formula or cereal, growth retardation, delayed wound healing, poor appetite, hair loss. Initially the lesion started on the hands and feet area then gradually spread to perioral (figure 1) and perianal (figure 2) areas. The child was born to consanguineous parents with normal vaginal delivery, with birth weight of $2 \mathrm{~kg}$. There was no history of any congenital abnormalities. There was no history suggestive of decreased calcium intake.

Cutaneous examination revealed multiple bilateral asymmetrical erythematous crusted plaque of varying diameters, ill-defined borders predominantly present over perianal perioral, perinasal, acral areas (figures $3 \mathrm{a}, 3 \mathrm{~b}$ ) scalp involvement with diffuse alopecia.

\author{
Corresponding Author: \\ Dr. Nilofer Ziauddin, \\ Flat No. 201, Wodden Sapphire Apartment, \\ Lalamma Gardens, Street No. 2, \\ Duddalguda, Manikonda, \\ Hyderabad-500089, Telangana, India. \\ E-mail: dr.nilofer25@gmail.com
}

DOI: $10.14260 /$ jemds/2020/318

Financial or Other Competing Interests: None.

How to Cite This Article:

Ziauddin N, Haneef NS, Nikhat. A rare case of acrodermatitis enteropathica in a one year old child. J. Evolution Med. Dent. Sci. 2020;9(17):1459-1463, DOI:
Submission 05-03-2020, Peer Review 15-04-2020, Acceptance 21-04-2020, Published 27-04-2020. 

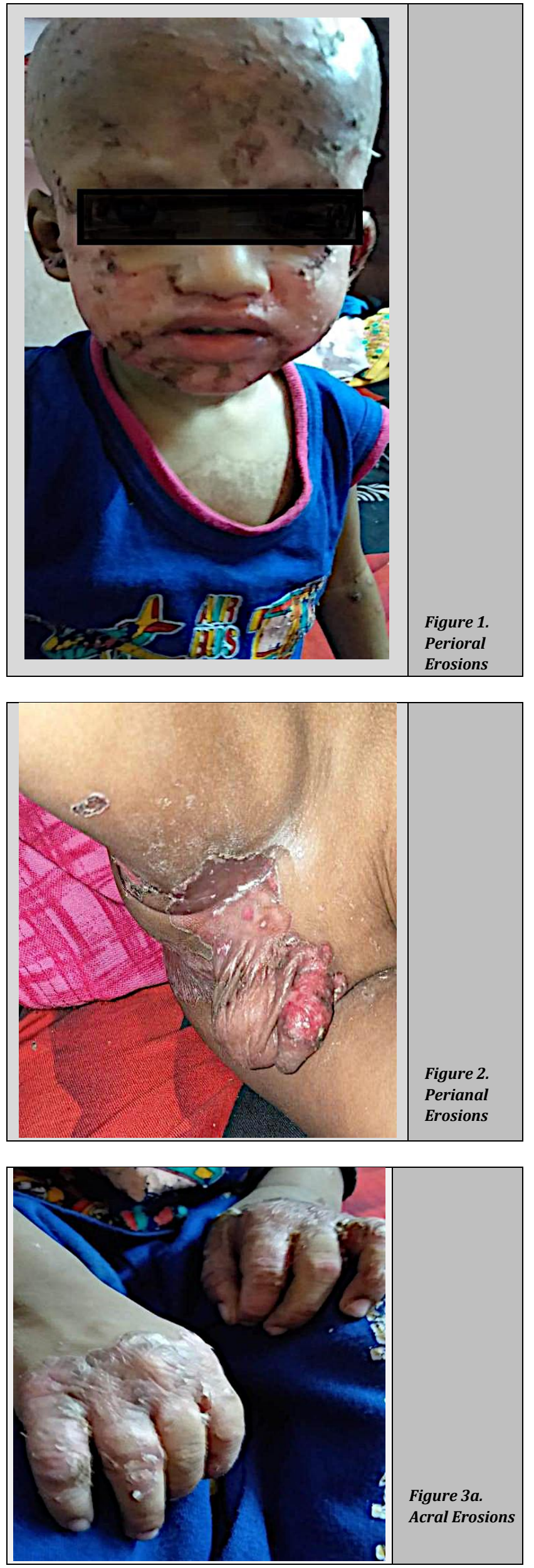

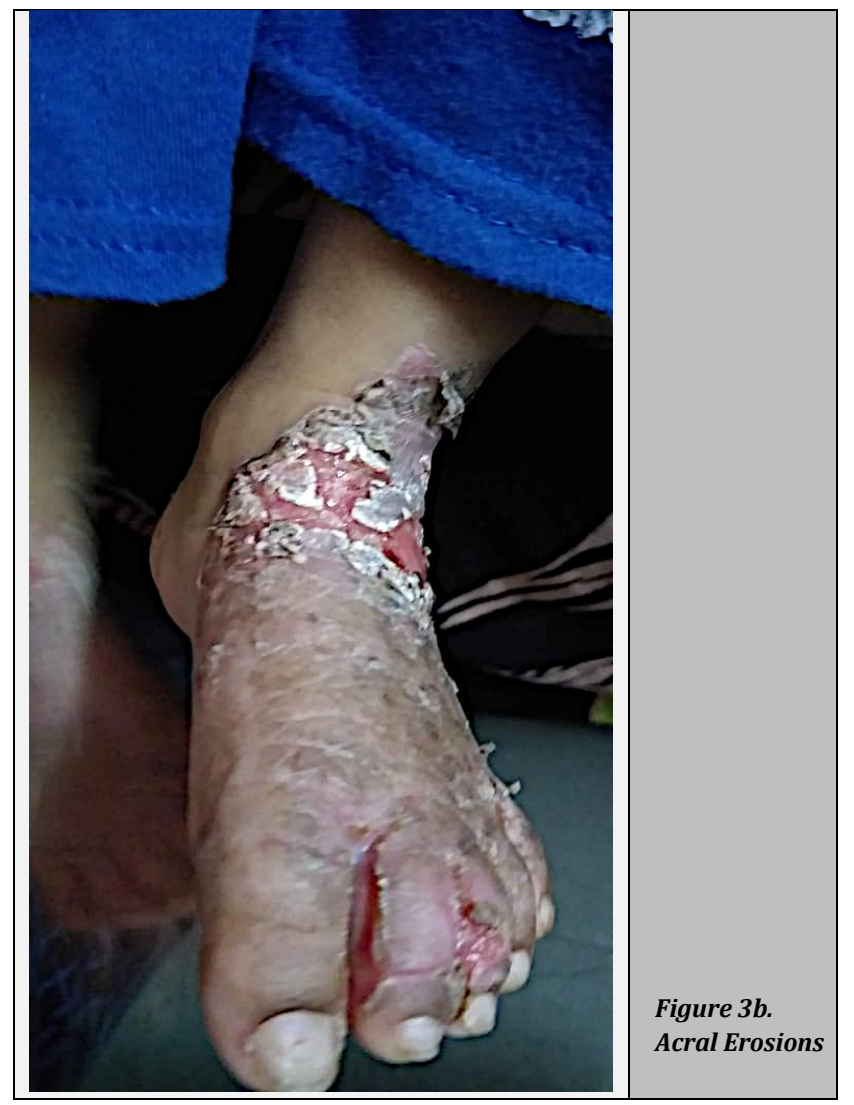

Differential diagnosis including epidermolysis bullosa simplexa, epidermolysis, acrodermatitis enteropathica, bullous pemphigoid, staphylococcal scalded skin syndrome, impetigo contagiosum were considered.

Skin biopsy showed partially ulcerated denuded epidermis with focally preserved malpighian layer, and few elongated rete ridges and mild spongiotic changes, seen. Underlying dermis showed perivascular mild lymphocytic infiltrate with occasional eosinophils and polymorphs (figure 5). Direct immunofluorescence study done using Fluorescein isothiocyanate (FITC) antibodies against IgG, IgM, IgA and C3c showed no immune deposits ruling out immunobullous diseases like epidermolysis bullosa simplexa, bullous pemphigoid. Serum zinc level was $46 \mathrm{mg} / \mathrm{dL}$ (normal: 70-110 $\mu \mathrm{g} / \mathrm{dL}$ ). Serum alkaline phosphatase was $78 \mathrm{u} / \mathrm{L}$ (normal: $115360 \mathrm{IU} / \mathrm{L})$.

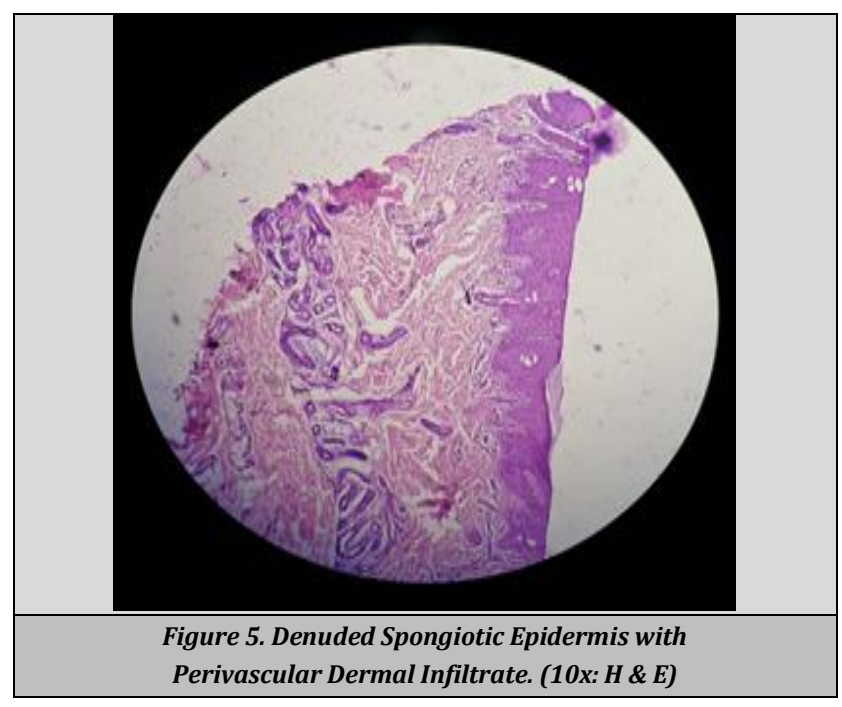




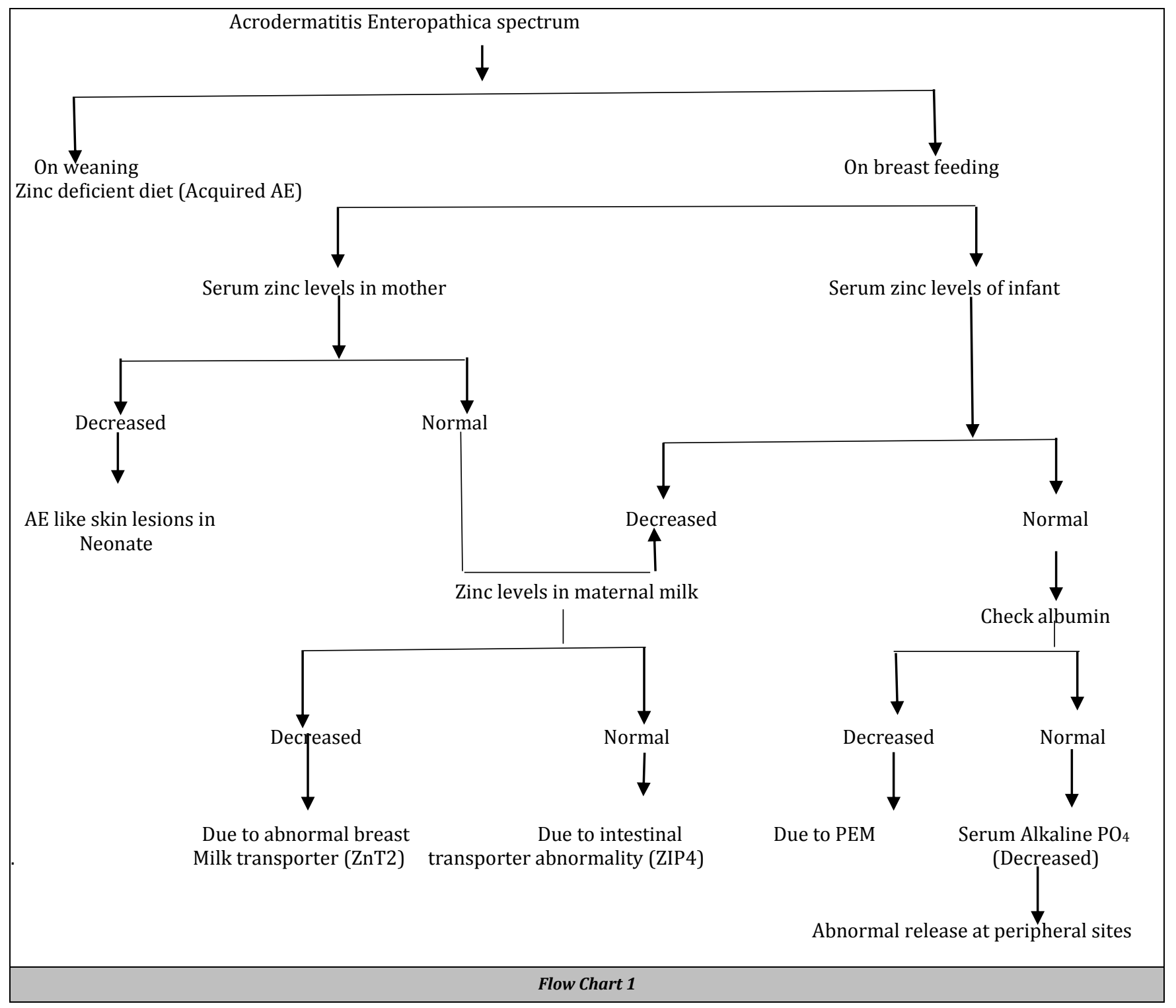

Zinc supplementation in the form of oral syrup containing $5 \mathrm{mg} / \mathrm{ml}$ of zinc sulphate was administered thrice daily for three months. Patient responded to treatment within 2 weeks. After 3 months patient showed good improvement with subsidence of all skin lesions and diarrhoea.

Patient developed frequent recurrences during the follow up period of one-half year on stopping the oral supplementation of zinc. Alopecia improved partially.

\section{DISCUSSION}

Zinc is an essential element in humans and plays an important role in growth and development.[4]It is estimated to affect about one in 500,000 children. Zinc deficiency in infants can be divided into three main subtypes. ${ }^{[4]}$ Primary zinc deficiency due to dietary inadequacy (older breastfed infants or toddlers without zinc-rich complementary foods)Acquired secondary deficiency in low birth weight and premature babies and various malabsorption syndromes Genetically based deficiency (includes classical $\mathrm{AE}$ and acquired zinc deficiency of lactogenic origin).
The classical is $\mathrm{AE}$ caused by a genetically determined defect in intestinal zinc transporters while, the acquired zinc deficiency of lactogenic origin is caused by defective zinc transporter in mother's milk [Flow Chart 1].[5]

ZIP4 is a low molecular weight pancreatic ligand, encoded by SLC39A4 gene (located on chromosomal region 8q24.3) which aids in zinc absorption in the intestine.[6] Alteration in the production of ZIP4 transporter is the basic genetic defect in classical $\mathrm{AE}$ us causing a partial block in intestinal zinc absorption. Classical, $\mathrm{AE}$ an autosomal recessive disorder and requires higher serum zinc level so as to overcome this partial block.[2,3] Diagnosis of classical AE as made in our case on the basis of decreased serum zinc levels, normal zinc level in breast milk and history of consanguinity in the parents. Initial signs and symptoms appear in early infancy in exclusively breastfed full term infants, as seen in our case, hence favour our diagnosis. ZnT2, the transporter present in maternal mammary glands is responsible for zinc secretion in human milk.[3] AE like disease due to a defect in ZnT2, also known as zinc deficiency of lactogenic origin, is characterized by early onset, with a brief and self-limiting course..$^{[7]}$

It clears when the children start their normal solid diet. This variant of $\mathrm{AE}$ is also known as transient neonatal zinc 
deficiency for the purpose of differentiation from classical AE. It has an autosomal dominant inheritance pattern.

Classic presentation of $\mathrm{AE}$ is of diarrhoea, skin rash with alopecia, extreme irritability and depression. Onset is approximately 1 to 2 weeks after weaning. Infants with $\mathrm{AE}$ have an erythematous, scaly, psoriasiform and sometimes vesiculopustular eruption located periorificially (i.e. around the mouth, eyes and genital areas). The finger flexural creases and the palms show characteristic flat greyish bullous lesions surrounded by red brown erythema. Skin lesions also appear on extremities. Refractory diaper dermatitis may be the presenting complaint. Secondary staphylococcal and candidal superinfections are very common, and do not respond to topical therapy unless the zinc deficiency is corrected. Paronychia and nail dystrophy can take place. Hair becomes dry and complete alopecia may develop. Diarrhoea starts early in the disease; however it is not a constant feature as reported by Naik et al.[8] Ocular involvement in the form of corneal changes consisting of superficial punctate lesions, nebulous subepithelial opacities and linear epithelial erosions have been reported. ${ }^{[9]} \mathrm{AE}$ has also been reported from both bottle and breast-fed infants. If left untreated, severe failure to thrive and death my ensue.[10,11]

Low levels of plasma zinc are pathognomonic of AE. Care must be taken while collecting blood samples. Samples should be collected in special acid-washed glass bulbs or plastic tubes, otherwise exogenous zinc present on ordinary glassware will contaminate the specimen. Mack et al,[12] reported a patient of $\mathrm{AE}$ with normal serum zinc levels in whom the diagnosis was confirmed by plasma phospholipid fatty acid and a small bowel biopsy. Other associated laboratory findings are low serum alkaline phosphatase, hypogammaglobulinemia, abnormal cell-mediated immunity or anergy.[13]

The actual metabolic error in has not yet been defined, but it is clearly a problem with intestinal absorption and/ or transport of zinc. Picolinic acid, a tryptophan metabolite, enhances the intestinal absorption of zinc which is deficient in AE.[14] Prasad et al,[15] have reported adolescents from the Middle-east with zinc deficiency which develops due to concurrent ingestion of high amounts of the zinc binding ligand, phytate present in high quantity in that area. Sandstorm et al,[16] suggested that the primary lesion in AE is in cellular defect in zinc metabolism rather than an impairment of zinc absorption.

The zinc derived from plant origin is less available for utilization and absorption due to high phytate and fibre content, than the zinc derived from animal product.[17]

The specific cutaneous manifestations of zinc deficiency may be in part due to zinc interaction with vitamin A metabolism. The interaction of zinc and vitamin A may be mediated via the enzyme retinol alcohol dehydrogenase, which converts photoactively inert retinol to the active retinaldehyde or by impairing synthesis of retinol binding protein.[18]

Hydroxyquinoline was found empirically to provide successful therapy in AE.[19] It most probably enhanced intestinal, zinc absorption. Zinc supplementation has a rapid and dramatic effect that reverses all cutaneous, gastrointestinal and neurological manifestations of the disease. Michaelsson,[20] was the first to use zinc in AE. Zinc can be administered as a sulfate $(22.5 \mathrm{mg}$ of elemental zinc/100 $\mathrm{mg}$ ). The zinc sulphate salt may sometimes act as an irritant to the GI tract and may precipitate bloody diarrhoea.[10] Bhargava et al[21],reported use of 'Jasad Bhasm' (which contains zinc) useful in two cases of AE. Zinc in a dose of about l- $2 \mathrm{mg} \mathrm{Kg} /$ day is useful to cure all clinical manifestations within 1-2 weeks. Zinc may need to be supplemented for life-time. However, it may show improvement with age.

\section{CONCLUSIONS}

We are reporting a rare case of one year old child with acrodermatitis enteropathica, which improved with oral zinc supplementation, but had frequent recurrences indicating genetic factor for zinc deficiency.

\section{Declaration of Patient Consent}

The authors certify that they have obtained all appropriate patient consent forms. In the form the patient(s) has/have given his/her/their consent for his/her/their images and other clinical information to be reported in the journal. The patients understand that their names and initials will not be published, and due efforts will be made to conceal their identity, but anonymity cannot be guaranteed.

\section{REFERENCES}

[1] Moynahan EJ, Barnes PM. Zinc deficiency and a synthetic diet for lactose intolerance. Lancet 1973; 301(7804):6767.

[2] Krebs NF. Update on zinc deficiency and excess in clinical pediatric practice. Ann Nutr Metab 2013; 62(Suppl 1):1929.

[3] Kasana S, Din J, Maret W. Genetic causes and genenutrient interactions in mammalian zinc deficiencies: Acrodermatitis enteropathica and transient neonatal zinc deficiency as examples. J Trace Elem Med Biol 2015; 29:47-62.

[4] Maverakis E, Fung MA, Lynch PJ, et al. Acrodermatitis enteropathica and an overview of zinc metabolism. J Am Acad Dermatol 2007; 56(1):116-24.

[5] Kaur S, Sangwan A, Dayal S, et al. Aplasia cutis congenita, group 5 without fetus papyraceus in two newborns. Indian J Dermatol Venereol Leprol 2016; 82(6):695-7.

[6] Nakano A, Nakano H, Nomura K, et al. Novel SLC39A4 mutations in acrodermatitis enteropathica. J Invest Dermatol 2003; 120(6):963-6.

[7] Sharma NL, Sharma RC, Gupta KR, et al. Self-limiting acrodermatitis enteropathica. A follow up study of three interrelated families. Int J Dermatol 1988; 27(7):485-6.

[8] Naik RPC, Baliga MM. Acrodermatitis enteropathica: case report. Ind J Dermatol Venereol Leprol 1981; 47(2):1179.

[9] Prabriputaloog A, Prakitrittranin W. Corneal involvement in acrodermatitis enteropathica. J Med Assoc Thailand 1992; 75(7):423-7. 
[10] Sharma NL, Sharma RC, Gupta KR, et al. Hypozincemia in infancy. Ind J Dermatol Venereol Leprol 1985; 51(5):25660.

[11] Lee MG, Hong KT, Kim JJ. Transient symptomatic zinc deficiency in a full-term breastfed infant. J Am Acad Dermatol 1990; 23(2 Pt 2):375-9.

[12] Mack D, Kolezke B, Cunnane S, et al. Acrodermatitis enteropathica with normal serum zinc levels: diagnostic value of small bowel biopsy and essential fatty acid determination. Gut 1989; 30(10):1426-9.

[13] Chandra RK. Acrodermatitis enteropathica: zinc levels and cell-mediated immunity. Pediatrics 1980; 66(5):78991.

[14] Moore ME, Moran JR, Greene HL. Zinc supplementation in lactating women: evidence for mammary control of zinc secretion. J Pediatrics 1984; 105(4):600-2.

[15] Prasad AS, Halstead JA, Nadimi M. Syndrome of iron deficiency anemia, hepato-splenomegaly, hypogonadism, dwarfism and geophagia. Am J Med 1961; 31(4):532-46.
[16] Sandstrom B, Cederblad A, Lindblad BS, et al. Acrodermatitis enteropathica, zinc metabolism, copper status, and immune function. Arch Pediatr Adolescent Med 1994; 148(9):980-5.

[17] Gharpade A, Reddy BSN. Zinc in dermatology. Ind J Dermatol Venereol Leprol 1982; 48(2):84-92.

[18] Lucky AW. Cutaneous manifestations of endocrine, metabolic and nutritional disorders. In: Schachnner LA, Hansen RC, eds. Pediatric dermatology. $2^{\text {nd }}$ edn. Churchill Livingstone 1995;2:1976-9.

[19] Shafi M, Shah SNA. Acrodermatitis enteropathica. Ind J Dermatol Venereol 1973;39(1):33-4.

[20] Sharma NL. Zinc - an update. Ind J Dermatol Venereol Leprol 1985;51(6):305-8.

[21] Bhargava RK, Garg P. 'Jasad Bhasm': a zinc salt supplement in acrodermatitis enteropathica, Ind J Dermatol Venereol Leprol 1979;45(3):221-5. 